\title{
Diseño de una formulación de jarabe de acetaminofeno para uso pediátrico
}

Design of an acetaminophen syrup formulation for pediatric use

DOI 10.5377pc.v0i16.8094

Clara Celenia Nuñez Guevara ${ }^{1}$

Irela Pérez Sanchez ${ }^{2}$

María Victoria Zelaya ${ }^{3}$

\section{RESUMEN}

En el presente trabajo se desarrolló una formulación de jarabe de acetaminofeno, con una dosis de $160 \mathrm{mg} / 5 \mathrm{ml}$, dirigida a formar parte del cuadro básico de medicamentos del Hospital Escuela Universitario de la Universidad Nacional Autónoma de Honduras. Realizándose un diseño estadístico experimental de mezcla D-optimal para determinar la combinación de cosolventes que permite solubilizar la dosis de acetaminofeno. Las variables independientes fueron el contenido de: agua, etanol, glicerina, sorbitol $70 \%$ y propilenglicol; mientras las respuestas fueron $\mathrm{pH}$ y contenido de fármaco disuelto. Todas las variables influyeron en la disolución del ingrediente farmacéuticamente activo (IFA); con una mayor incidencia del etanol y el propilenglicol. Las mezclas óptimas lograron disolver inicialmente la dosis del IFA, pero luego de quince días ocurrió la recristalización del fármaco. Dada esta situación, se incorporó $7 \%$ de polivinilpirrolidona (K-30), ello garantizó la solubilidad del acetaminofeno, logrando una apariencia adecuada. Posteriormente se realizaron pruebas sensoriales evaluadas por jueces 60 no entrenados que permitieron seleccionar al sabor fresa $(0,2 \%)$ y el color rojo FD\&C $40(0,4 \%)$ como los correctores de sabor y color, respectivamente. Además se realizó la validación del método analítico por Cromatografía Líquida de Alta Resolución establecido en la USP 35. El método resultó ser lineal, preciso y selectivo para el análisis del acetaminofeno en la formulación propuesta, pero no cumplió con el parámetro de exactitud. Finalmente se efectuó el control de

1 Profesora del Departamento de Tecnología Farmacéutica y Asistente Técnico de Laboratorio UNAH: clara.nunez@unah.edu.hn

2 Universidad de la Habana Cuba, Profesora de la Maestría en Tecnología y Control de Medicamentos. irelaperez@infomed.sld.cu

${ }^{3}$ Director Laboratorio Industrial José Manuel Villeda Morales, UNAH, Profesor Titular IV, maria.zelaya@unah.edu.hn 
calidad químico y tecnológico de las preparaciones elaboradas, a los 0 y 90 días y el análisis microbiológico preliminar cumpliendo con las especificaciones establecidas.

Palabras clave: Jarabe, acetaminofeno, formulación, diseño, medicamentos

\section{ABSTRACT}

In the present work, a formulation of acetaminophen syrup was developed, with a dose of $160 \mathrm{mg} / 5 \mathrm{ml}$, aimed at forming part of the basic table of medicines of the University School Hospital of the National Autonomous University of Honduras. An experimental statistical design of D-optimal mixture was carried out to determine the combination of cosolvents that allows solubilizing the dose of acetaminophen. The independent variables were the content of: water, ethanol, glycerin, sorbitol $70 \%$ and propylene glycol; while the responses were $\mathrm{pH}$ and dissolved drug content. All the variables influenced the dissolution of the pharmaceutically active ingredient (IFA); with a higher incidence of ethanol and propylene glycol. The optimal mixtures were able to initially dissolve the IFA dose, but after fifteen days the recrystallization of the drug occurred. Given this situation, $7 \%$ of polyvinylpyrrolidone (K-30) was incorporated, this guaranteed the solubility of acetaminophen, achieving an adequate appearance. Subsequently, sensory tests evaluated by untrained judges 60 were performed, which allowed to select the strawberry flavor $(0.2 \%)$ and the red color FD \& C 40 $(0.4 \%)$ as the flavor and color correctors, respectively. In addition, the analytical method was validated by High Resolution Liquid Chromatography established in USP 35. The method was linear, precise and selective for the analysis of acetaminophen in the proposed formulation, but did not comply with the accuracy parameter. Finally, the chemical and technological quality control of the elaborated preparations was carried out, at 0 and 90 days and the preliminary microbiological analysis fulfilling the established specifications.

Keywords: Syrup, acetaminophen, formulation, design, medicines. 


\section{INTRODUCCIÓN}

Según el diario oficial La Gaceta (2012), a partir del 10 de julio de 2012 según decreto ejecutivo PCM-024-2012, el Gobierno de Honduras mediante la Secretaria de Salud Pública (SSP) traspasa la administración y control del Hospital Escuela Universitario (HEU) a la Universidad Nacional Autónoma de Honduras (UNAH). En el convenio marco (UNAH-SSP) se estableció "...La Secretaria y La Universidad reconocen la salud como un bien público, un derecho humano fundamental y una responsabilidad del Estado..." (Congreso Nacional. Acuerdos y Leyes., 2012, pág. 9) Este derecho está íntimamente relacionado al consumo de productos de calidad, seguridad y aptos para el fin previsto, mediante los servicios que ofrece el HEU.

La Universidad Nacional Autónoma de Honduras como ente administrativo del HEU enfrenta uno de los mayores retos, como es, brindar a sus usuarios medicamentos de calidad a través de proveedores fiables. Para ello pretende, como parte de su administración contar con un Laboratorio Farmacéutico Universitario, el cual proveerá de medicamentos pertenecientes al cuadro básico de medicamentos, como lo menciona el periódico Presencia Universitaria (Universidad Nacional Autonoma de Honduras., 2014)

Actualmente, en Honduras el acetaminofeno está siendo muy utilizado, debido a un brote epidémico de chikungunya. Los medicamentos que contienen este fármaco constituyen productos de primera elección para el tratamiento de la enfermedad. Ello confirma la importancia de elevar la disponibilidad de estos medicamentos. (Gobierno de la República de Honduras. Secretaria de Salud., 2014)

Tomando en cuenta los antecedentes relacionados, se decidió llevar a cabo una investigación que permitiera desarrollar una formulación de jarabe de acetaminofeno para uso pediátrico con una dosificación de $160 \mathrm{mg} / 5 \mathrm{ml}$.

\section{METODOLOGÍA}

\section{Diseño}

La presente investigación tiene un enfoque cuantitativo, con un diseño de tipo experimental, ya que se modifican de forma intencional las variables independientes 
(proporción de cosolventes) para analizar las consecuencias generadas sobre las variables dependientes ( $\mathrm{pH}$ y cantidad de IFA disuelto).

\section{Población y muestra}

Lotes de jarabe de acetaminofeno elaborados, utilizando acetaminofeno, glicerina, metilparabeno y propilparabeno (Proveedor Corporación Quirsa, El Salvador), Polivinilpirrolidona (K-30), sorbitol $70 \%$, propilenglicol, sacarina sódica, alcohol metílico, color rojo FD\&C 40 y sabor freza (Proveedor Distribuidora del Caribe, S.A.), sacarosa (Proveedor Ingenio Azucarero El cañal) y agua purificada (Proveedor Facultad de Química y Farmacia UNAH). Utilizando estándar de acetaminofeno obtenido de Anqiu Lu'An Pharmaceutical Co., Ltd. (Proveedor Corporación Quirsa, El Salvador).

\section{Entorno}

El diseño de la formulación de acetaminofeno jarabe se realizó en el espacio físico del laboratorio 205 y el desarrollo del método analítico de cuantificación en el laboratorio de equipos especiales, ambos ubicados en la Facultad de Química y Farmacia de la UNAH.

\section{Intervenciones y análisis estadístico}

Evaluación de la materia prima de acetaminofeno: Se empleó acetaminofeno materia prima correspondiente al lote 1551364 de Anqiu Lu'An Pharmaceutical Co., Ltd. Efectuándose los análisis necesarios que permitan comprobar la calidad de dicha materia prima, basándose en lo descrito en la USP 35 del 2012.

Selección de cosolventes: Se realizó un diseño de mezcla cuadrático D-optimal con la ayuda del programa Design Expert 8 (DX-8), para evaluar la influencia de los co-solventes en la solución, obteniendo un diseño con 20 muestras. Las variables estudiadas fueron: agua ( $57-84 \%)$, etanol $(0-3 \%)$, glicerina ( $5-20 \%)$, sorbitol 70 $\%(5-20 \%)$ y propilenglicol ( $5-20 \%)$; para un total de mezcla a evaluar del $100 \%$. Las variables dependientes a estudiar fueron $\mathrm{pH}$ y contenido de acetaminofeno disuelto. Además, se evaluó desde el punto de vista tecnológico la apariencia de la solución obtenida recién elaborada y transcurridos ocho días.

Optimización estadística del diseño: La optimización estadística del diseño se realizó de forma numérica. Para ello se utilizó el programa estadístico DX-8. Las variables independientes estudiadas (porcientos de agua, etanol, glicerina y propilenglicol) se 
mantuvieron en el rango de valores estudiados, con una importancia media (tres). En el caso del sorbitol $70 \%$, se propuso que se mantuviera en el nivel mínimo, con una importancia de cuatro. Con relación a las variables dependientes, se consideró el pH en el rango de valores obtenidos, con una importancia media (tres) y para el porciento de paracetamol disuelto se propuso el máximo nivel, e importancia máxima (cinco). Las mezclas seleccionadas como óptimas se ordenaron según el valor de desirabilidad calculado por el programa, lo que permitió confirmar la probabilidad de tener éxito durante la realización práctica del experimento.

Elaboración de muestras: La elaboración del jarabe se realizó a escala de laboratorio, por triplicado, con un volumen inicial de $100 \mathrm{ml}$ por cada réplica. La agitación se realizó de forma manual con la ayuda de una varilla de agitación. La filtración se realizó por gravedad, teniendo como medio filtrante papel de filtración rápida.

Las muestras de jarabe obtenidas se caracterizaron mediante las determinaciones de la apariencia de la solución, el pH y el contenido de acetaminofeno disuelto.

Selección de correctores de propiedades psicofisiológicas: El sabor más adecuado para el jarabe se determinó mediante una evaluación sensorial. Se ensayaron tres variantes de sabor, utilizando sabor limón $0.3 \%$, sabor fresa $0.3 \%$ y una muestra de jarabe sin sabor que sirvió como blanco. Las variantes diseñadas fueron evaluadas por sesenta jueces no entrenados; los cuales degustaron $2 \mathrm{ml}$ de cada muestra de jarabe preparado, y respondieron una encuesta correspondiente a una prueba de preferencia por ordenamiento siguiendo una escala hedónico.

Los resultados de las encuestas aplicadas fueron analizados desde el punto de vista estadístico. Para ello a cada categoría aplicada en la encuesta se le asignó una evaluación numérica.

Posteriormente, se aplicó la prueba no paramétrica de Kruskal - Wallis para determinar si existen diferencias significativas entre las muestras analizadas, teniendo un nivel de significación ( $\nabla$ ) igual a 0,05. En caso de observar diferencias significativas entre las muestras, se aplicó la prueba de rango múltiples de Duncan y de esta forma de estableció que sabores eran diferentes entre sí, en cuanto a la preferencia de los jueces y cuál de ellos resultaba el de mayor aceptación. Todo el análisis estadístico se realizó a través del programa Statgraphics 5.1.

Tomando en cuenta el sabor seleccionado, se utilizó el color rojo para la formulación diseñada. Para establecer la proporción de colorante que debía estar en la formula- 
ción se procedió a aplicar una evaluación sensorial. Se ensayaron tres concentraciones de colorante $0,2 \%, 0,3 \%$ y $0,4 \%$, se prepararon muestras de jarabe con dichas proporciones de color, las cuales fueron evaluadas de la misma forma descrita anteriormente para la selección del saborizante.

Validación del método analítico de cuantificación: Se tomó como punto de partida el procedimiento por cromatografía líquida de alta resolución descrito en la USP 35 para la solución oral de acetaminofeno.

Para el desarrollo del método analítico se utilizó un Cromatógrafo Líquido de Alta Resolución (HPLC) marca Shimadzu, modelo Prominence, con un detector de arreglo de diodos SPD-M20A, una bomba LC20-AT, horno de columna CTO-A20, inyector automático SIL-20A y el software EZ start para la recolección de datos cromatográficos. Las condiciones cromatográficas trabajadas fueron una columna eclipse XDB-C18, $5 \mu \mathrm{m}, 150 \times 4.6 \mathrm{~mm}$, velocidad de flujo de fase móvil de $1,5 \mathrm{ml} / \mathrm{min}$ y volumen de inyección de $10 \mu \mathrm{m}$ para solución estándar y muestra. Longitud de onda de $243 \mathrm{~nm}$. La fase móvil utilizada consistía de mezcla desgasificada de agua y metanol (3:1) con solventes calidad HPLC marca J.T. Baker, adquiridos en Labhospy, S de R.L. Tegucigalpa.

Los parámetros evaluados para validar este método analítico fueron establecidos en función del propósito con que fue desarrollado (método de control de pruebas cuantitativas del contenido de IFA). Como lo indican las autoridades regulatorias (RTCA. Comités Técnicos de Normalización y de Reglamentación Técnica, 2006) y (Harmonised Tripartite Guideline (ICH), 1996), este tipo de proceder pertenece a la categoría I de procedimientos analíticos, por tanto se evaluó la linealidad, rango de trabajo, exactitud, precisión y especificidad. Los cálculos estadísticos para demostrar la validez de dicho método se realizaron según lo establecido en las normas de validación de los métodos de análisis y utilizando el programa ofimático Microsoft Excel para facilitar el procesamiento de los resultados.

\section{RESULTADOS Y DISCUSIÓN}

Caracterización de la materia prima acetaminofeno: Los resultados generales obtenidos durante la caracterización del fármaco como ser descripción, solubilidad, valoración e identificación realizándose mediante 2 técnicas analíticas (cromatografía en capa delgada y espectrofotometría ultravioleta), coincidió con las especificaciones establecidas en la USP 35 del 2012. 
Selección del co-solvente: En los veinte experimentos evaluados se lograron formar soluciones. De forma particular, aquellos ensayos donde el etanol estaba en concentraciones mínimas, con niveles de propilenglicol y/o glicerina bajos, mostraron mayor dificultad para lograr la solubilidad de toda la masa de IFA adicionada.

Con respecto al pH, se observó muy poca variación entre los diferentes ensayos del diseño, los valores oscilaron entre 3,8 y 5,3; valores que resultan adecuados para una preparación líquida oral y están comprendidos dentro del rango de $\mathrm{pH}$ de mayor estabilidad del acetaminofeno, según indica la literatura consultada (USP 35 del 2012).

La apariencia de las soluciones indicó la presencia de cristales de IFA no disueltos al finalizar el proceso de filtración en aquellas experiencias que mostraron más dificultad para lograr la disolución del fármaco. Este comportamiento demostró que se el sistema formado se encontraba muy cerca de la concentración de saturación. En otras preparaciones, donde los niveles de glicerina y sorbitol se encontraron en los niveles medios; si bien en los momentos iniciales se observó una preparación homogénea y traslúcida, con el transcurrir de los días se formaron algunos cristales que le dieron opalescencia a la preparación. Este resultado evidencia que la mezcla de co-solventes formulada no era suficiente para garantizar la solubilidad de la masa de fármaco que se incorporó al sistema.

En correspondencia con lo anterior, los porcientos de acetaminofeno disueltos mostraron una mayor variación, alcanzando resultados positivos (cercanos a $100 \%$ ) solo en aquellos ensayos donde se mantuvo una apariencia adecuada, aunque al finalizar el ensayo (ocho días después de preparadas las muestras) se observaron pequeños cristales. Ese es el caso de ensayos que coinciden en mostrar los niveles en mínimos de agua, medios a bajos de glicerina y sorbitol, medios a altos de etanol y elevados de propilenglicol.

Optimización del diseño: Tomando en cuenta los resultados anteriores, se procedió a la optimización numérica del diseño. Como resultado se obtuvieron 11 posibles muestras con diferentes niveles de desirabilidad, de las cuales se seleccionaron 2 de las mezclas propuestas, por ser, dentro del grupo de soluciones que contienen etanol, las que mantienen los niveles mínimos con una desirabilidad superior al $90 \%$ y permiten obtener un contenido de paracetamol disuelto cercano al $100 \%$. Se hizo hincapié en mantener un mínimo de etanol dada la alta solubilidad del fármaco en este solvente. Luego se elaboraron las mezclas seleccionadas bajo las mismas condiciones que se utilizaron para la elaboración de las soluciones del diseño anterior. 
Desde el punto de vista tecnológico ambas mezclas de solventes seleccionadas mostraron valores de $\mathrm{pH}$ muy similares y dentro del rango establecido para este producto en la literatura oficial (3,8 - 6,1 según USP 35). Con relación a los niveles de fármaco disuelto, ambas mezclas lograron disolver la dosis añadida, alcanzando niveles de acetaminofeno cercanos al $100 \%$.

Al analizar las características organolépticas de estas mezclas recién preparadas se constató que las mismas constituían líquidos, traslúcidos, brillantes, transparentes y con ausencia total de cristales. Sin embargo, transcurridos 72 horas posteriores a la preparación, las tres réplicas de la muestra 6 presentaron en el fondo del recipiente pequeños cristales que indicaban la precipitación del IFA. De esta forma quedó demostrado que esta mezcla de solventes resultaba insuficiente para mantener disuelta la dosis de acetaminofeno necesaria y por tanto quedó descartado su uso.

A partir de los resultados anteriores se decidió continuar la observación de las réplicas de la única muestra que se encontraba solubilizada, pero transcurridos 15 días, se observó la formación de cristales en el fondo del recipiente que la contenía, indicando que el fármaco recristalizaba. Ante este resultado se decidió buscar otra metodología que permitiera garantizar la solubilidad de la dosis de acetaminofeno deseada y con un mínimo de etanol en la preparación.

Efecto del polímero polivinilpirrolidona sobre la solubilización del acetaminofeno: Tomando en cuenta los resultados obtenidos en el análisis anterior con la mezcla de co-solventes, se decidió elaborar una nueva preparación en la que se incluyeran además de los co-solventes etanol, glicerina y propilenglicol; el polímero polivinilpirrolidona (PVP), material que posee una reconocida capacidad para mejorar la solubilidad de fármacos poco solubles (Wen, Morris, \& Park 2005; Iwata \& Ueda, 1996) En este particular se aumentó los niveles de glicerina en aras de mejorar el sabor, minimizar los niveles de sacarosa y con ello poder disminuir los niveles de agua, este último es el solvente donde el acetaminofeno muestra baja solubilidad. También se incorporó sacarina sódica para acentuar el sabor dulce, debido a la disminución en el contenido de sacarosa.

Durante la elaboración del jarabe se logró disolver la dosis de fármaco prevista. Como resultado final, se obtuvo una solución, clara, brillante, transparente y traslúcida, de color rojo, sabor dulce y libre de cristales precipitados. Estas muestras se mantuvieron al abrigo de la luz y a una temperatura de $30^{\circ} \mathrm{C}$, aproximadamente. Bajo esas condiciones se estuvo observando la apariencia de la solución durante 30 días 
y todo el tiempo de ensayo mantuvo las mismas características iniciales en sus tres réplicas.

Trascurridas 24 horas posteriores a la preparación, se determinó el pH de la formulación el cual fue de 4,84 $\pm 0,04$. Este resultado, puede considerarse positivo, debido a que se encuentra dentro del rango de valores recomendado para este producto en la USP 35 (pH entre 3,8 y 6,1). Este resultado se comprobó al finalizar el ensayo y se alcanzó un resultado similar, lo que demuestra la estabilidad de la solución en cuanto al $\mathrm{pH}$.

Con relación al contenido de paracetamol disuelto en la preparación, se constató durante el análisis que dicho parámetro alcanzó un valor promedio de 97,11\% 0,11. De esta forma se demostró que el sistema propuesto era capaz de disolver la dosis de acetaminofeno prevista. Tomando en cuenta los resultados obtenidos, se decidió continuar el estudio con esta formulación, la cual se muestra en la tabla I. El método de preparación utilizado se describe en el diagrama de flujo de la figura 1.

Selección de los correctores de las propiedades psicofisiológicas: Dado que la formulación objeto de estudio es una solución oral y que la misma está dirigida a la población infantil, resultó necesario proporcionarle un sabor agradable. Debido a su composición, se denota un sabor dulce; sin embargo no resultaba suficiente para enmascarar el sabor amargo del acetaminofeno. Es por ello que se decidió utilizar saborizantes que mejoraran esta propiedad psicofisiológica. Seleccionándose el sabor freza $0,2 \%$ y el color rojo FD\&C 40 al 0,4 \% como los correctores indicados.

Procedimiento de elaboración: Tomando como punto de partida la formulación desarrollada y el procedimiento utilizado para elaborar la misma se elaboraron tres lotes de jarabe, siguiendo el procedimiento que se muestra en la figura 1.

Durante el proceso de elaboración se logró disolver la dosis de acetaminofeno establecida, no apreciándose la formación de cristales durante todo el proceso. De esta forma se demostró la reproducibilidad del proceso desarrollado y la factibilidad para desarrollar el mismo. El producto obtenido fue envasado y acondicionado. El mismo se mantuvo en un lugar fresco a una temperatura aproximada de $30^{\circ} \mathrm{C}$, protegido de la incidencia directa de la luz. 
Tabla I. Formulación para el jarabe de acetaminofeno incorporando co-solventes y el polímero polivinilpirrolidona.

\begin{tabular}{|l|l|c|}
\hline Componente & Función & Cantidad \\
\hline Acetaminofeno & Fármaco & $3,20 \mathrm{~g}$ \\
\hline Polivinilpirrolidona (K-30) & Solubilizante & $7,00 \mathrm{~g}$ \\
\hline Alcohol etílico & Co-solvente & $3,00 \mathrm{~mL}$ \\
\hline Glicerina & Co-solvente & $20,00 \mathrm{~mL}$ \\
\hline Propilenglicol USP & Co-solvente & $20,00 \mathrm{~mL}$ \\
\hline Sacarosa & Edulcorante & $10,00 \mathrm{~g}$ \\
\hline Sacarina Sódica & Edulcorante & $0,60 \mathrm{~g}$ \\
\hline Metilparabeno & Conservante & $0,18 \mathrm{~g}$ \\
\hline Propilparabeno & Conservante & $0,02 \mathrm{~g}$ \\
\hline Color rojo FD\&C 40 & Colorante & $0,40 \mathrm{~g}$ \\
\hline Sabor fresa & Saborizante & $0,20 \mathrm{~mL}$ \\
\hline Agua purificada c.s.p. & Solvente & $100,00 \mathrm{~mL}$ \\
\hline
\end{tabular}

Fuente: Creación propia

Figura 1. Flujo tecnológico de elaboración de jarabe de acetaminofeno con PVP
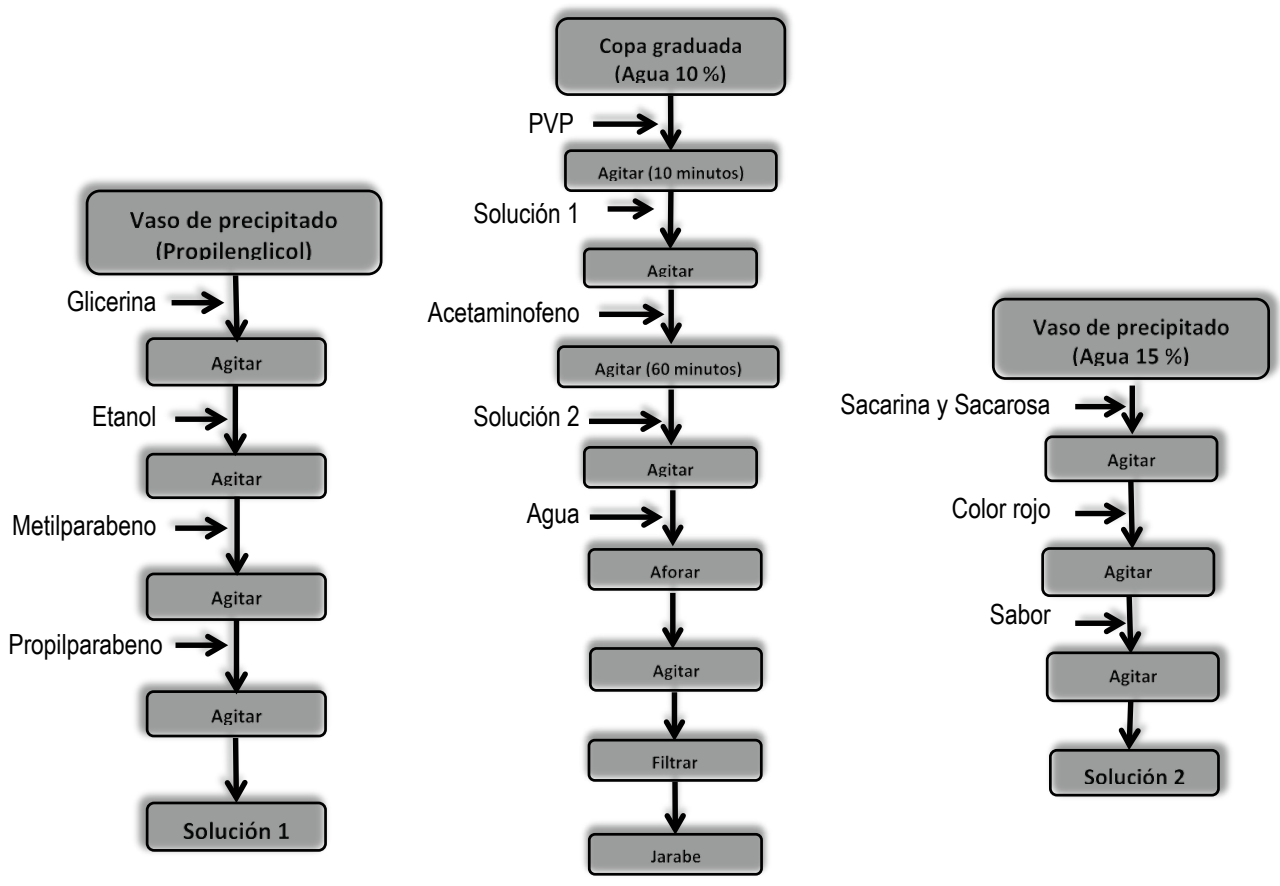

Fuente: Creación propia 
Validación del método analítico: Se obtuvieron resultados en cuanto a la validación del método analítico que permiten afirmar que el método utilizado es lineal, preciso y específico, logrando cumplir con las especificaciones descritas por la USP 35 para estos parámetros, no así para la exactitud.

Al realizar el análisis de una muestra de placebo de la formulación en estudio, se observó en el cromatograma un pequeño pico a aproximadamente 1,8 minutos, sin mostrar interferencia con la sustancia de interés a los 3,2 min de elución del estándar y muestras analizadas, como lo muestra la figura 2.

Figura 2. Cromatogramas analizados con HPLC con detector de arreglo de diodos. Nota: A Solución estándar, B Muestra de formulación en estudio y C Placebo.

A

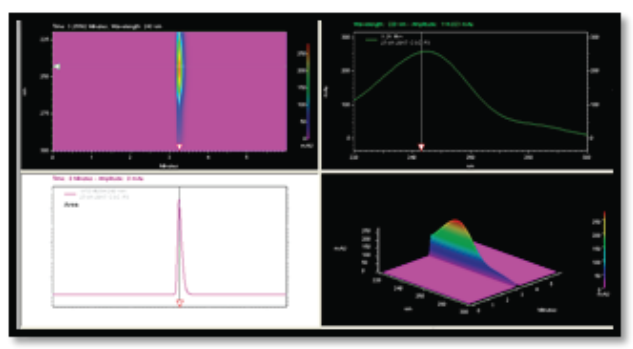

B

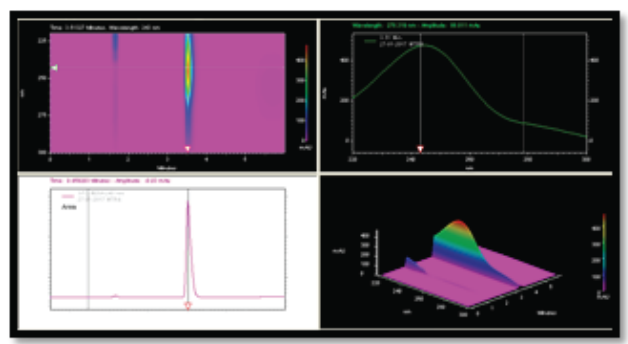

C

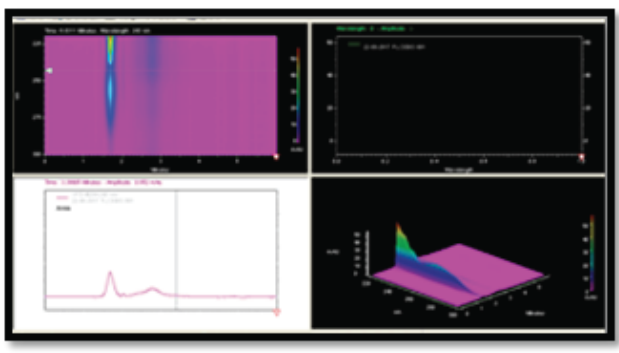

Fuente: Cromatógrafo líquido de alta resolución, marca Shimadzu.

Control de la calidad del jarabe: Los tres lotes elaborados fueron sometidos al control de calidad para verificar el cumplimiento de las especificaciones de calidad establecidas para este producto en la literatura especializada. La tabla II muestra los obtenidos. Como se puede constatar los tres lotes mostraron resultados similares. 


\section{Tabla II. Resultados del control de la calidad del jarabe producido a escala piloto}

\begin{tabular}{|c|c|c|c|c|}
\hline \multirow{2}{*}{ Pruebas } & \multirow{2}{*}{ Criterios de Aceptación } & \multicolumn{3}{|c|}{ Resultados } \\
\hline & & Lote 1 & Lote 2 & Lote 3 \\
\hline Descripción & $\begin{array}{l}\text { Líquido homogéneo ligeramente viscoso, color } \\
\text { rojo brillante traslucido, olor y sabor a fresa } \\
\text { dulce. }\end{array}$ & Cumple & Cumple & Cumple \\
\hline $\mathrm{pH}$ & $3,8-6,1$ & 4,83 & 4,90 & 4,88 \\
\hline Densidad relativa & --- & $1,18 \mathrm{~g} / \mathrm{mL}$ & $1,18 \mathrm{~g} / \mathrm{mL}$ & $1,18 \mathrm{~g} / \mathrm{mL}$ \\
\hline Viscosidad & --- & $120 \mathrm{cP}$ & $119 \mathrm{cP}$ & $121 \mathrm{cP}$ \\
\hline Identificación & $\begin{array}{c}\text { Correspondencia en el tiempo de retención del } \\
\text { pico principal en el cromatograma de muestra y } \\
\text { estándar, }\end{array}$ & Cumple & Cumple & Cumple \\
\hline Valoración & $90,0 \%-110,0 \%$ & $99,83 \%$ & $101,24 \%$ & $98,03 \%$ \\
\hline
\end{tabular}

Nota: cP es unidad en centipoise.

Fuente: Creación propia

La identificación permitió constatar la presencia del acetaminofeno como analito en las muestras tratadas, al obtener la señal del pico de elución con tiempos de retención muy similares a los obtenidos con el análisis del estándar de referencia de este IFA (tiempo de retención de la muestra 3,2529 min; tiempo de retención del estándar $3,2861 \mathrm{~min})$.

El análisis cuantitativo permitió demostrar que los lotes elaborados contenían más del $99 \%$ de la dosis declarada, valores que se encuentran dentro del rango establecido en la literatura especializada para este tipo de preparación. Este resultado garantiza que toda la dosis de fármaco se mantiene en solución y no ocurrió perdida por precipitación de la misma.

\section{CONCLUSIONES}

1. El acetaminofeno, producido por la empresa Anqiu Lu'An Pharmaceutical Co, Ltd, de procedencia China, cumplió con las exigencias establecidas en la USP 35, por lo que se consideró apto para ser empleado en la elaboración de medicamentos. 
2. El diseño estadístico experimental de mezcla demostró que las concentraciones de agua, etanol, glicerina, sorbitol $70 \%$ y propilenglicol tienen una marcada influencia sobre la solubilidad del acetaminofeno, siendo el etanol y el propilenglicol los de mayor incidencia. No obstante, resultaron insuficientes para mantener el IFA disuelto en la dosis recomendada.

3. La incorporación de un $7 \%$ de polivinilpirrolidona al sistema que contenía: etanol $3 \%$; glicerina $20 \%$ y propilenglicol $20 \%$, permitió garantizar la solubilidad del acetaminofeno en solución acuosa.

4. Las pruebas de evaluación sensorial realizadas a la formulación elaborada permitieron seleccionar el sabor fresa $(0,2 \%)$ y el color rojo FD\&C $40(0,4 \%)$ como los correctores de sabor y color, respectivamente, más adecuados para enmascarar las propiedades psicofisiológicas en la preparación de jarabe diseñado.

5. El método cromatográfico de alta resolución aplicado en el análisis del jarabe de acetaminofeno formulado, logró satisfacer las exigencias establecidas en los parámetros linealidad, precisión y especificidad; no así para la exactitud.

6 . Los lotes de jarabe de acetaminofeno elaborados, cumplieron de forma satisfactoria con el control de calidad químico y tecnológico, así como microbiológico preliminar, al satisfacer las exigencias establecidas en la USP 35 del 2012.

\section{AGRADECIMIENTOS}

A las autoridades de la Facultad de Química y Farmacia por la oportunidad de proporcionar el espacio físico y los equipos necesarios para el desarrollo de la investigación. Al Dr. Henry Ponce MSc., por apoyar con sus conocimientos y experiencia en el área analítica durante el proceso de validación del método utilizado.

\section{REFERENCIAS BIBLIOGRÁFICAS}

Congreso Nacional. Acuerdos y Leyes. (13 de Julio de 2012). Decreto ejecutivo PCM-024-2012. Convenio Interinstitucional entre órganos de la Administración pública para la asignación de la dirección, administración y funcionamiento del hospital-escuela a la Universidad Nacional. La Gaceta Diario Oficial de la Republica de Honduras(32,871), pág. 9. Recuperado el 15 de Junio de 2015

Gobierno de la República de Honduras. Secretaria de Salud. (2014). Guía de Manejo Clínico de Fiebre Chikungunya. Recuperado el 20 de Julio de 2015, de http://www.salud.gob.hn/noticias/boletin\%20mayo2014/guiamanejoclinicochikv.pdf 
Harmonised Tripartite Guideline (ICH). (6 de Noviembre de 1996). Validation of Analytical Procedures: Text and Methodology Q2 (R1). 17.

Jato, J. L. (2001). Tecnología Farmaceutica (Vol. 2). Madrid, España: Síntesis S.A. Obtenido de http://es.slideshare.net/marcovinicioroblesaguilar/formas-farmacuticas-jos-luis-vila-ja to

Nair, J. G. (2003). Remington Farmacia (20 ed., Vol. 1). (A. R. Gennaro, J. P. Reminton, \& S. Belluci, Edits.) Buenos Aires, Argentina: Medica Panamericana. Obtenido de https://books.google.hn/books?id=Av4llsyH-qcC\&

RTCA. Comités Técnicos de Normalización y de Reglamentación Técnica. (2006). Reglamento Técnico Centroamericano. Productos Farmacéuticos. Validación de Métodos Analíticos para la Evaluación de la Calidad de los Medicamentos, 8. Guatemala.

Universidad Nacional Autonoma de Honduras. (21 de Agosto de 2014). Presencia Universitaria. El periodico de la reforma. (C. Alvarenga, Ed.) Recuperado el 17 de Julio de 2015, de https://presencia.unah.edu.hn/salud/articulo/unah-crearalaboratorio-para-fabricarmedicinas-.

USP. Farmacopea de los Estados Unidos de América (35 ed., Vol. 2). (2012). Washington D.C, Estados Unidos de América.

Wen , H., Morris, K., \& Park, K. (Octubre de 2005). PubliMed. Recuperado el 30 de Agosto de 2017, de Study on the interactions between polyvinylpyrrolidone (PVP) and acetaminophen crystals: partial dissolution pattern change: https://www.ncbi.nlm.nih.gov/pub$\mathrm{med} / 16136578$ 